\title{
Richness-mass relation self-calibration for galaxy clusters ${ }^{\star}$
}

\author{
S. Andreon ${ }^{1}$ and J. Bergé ${ }^{2}$ \\ 1 INAF - Osservatorio Astronomico di Brera, via Brera 28, Milano, Italy \\ 2 ETH Zurich, Department of Physics, Wolfgang Pauli Strasse 27, 8093 Zurich, Switzerland \\ e-mail: stefano.andreon@brera.inaf.it
}

Received 27 July 2012 / Accepted 20 September 2012

\begin{abstract}
This work attains a threefold objective: first, we derived the richness-mass scaling in the local Universe from data of 53 clusters with individual measurements of mass. We found a $0.46 \pm 0.12$ slope and a $0.25 \pm 0.03$ dex scatter measuring richness with a previously developed method. Second, we showed on a real sample of $2500.06<z<0.9$ clusters, most of which are at $z<0.3$, with spectroscopic redshift that the colour of the red sequence allows us to measure the clusters' redshift to better than $\Delta z=0.02$. Third, we computed the predicted prior of the richness-mass scaling to forecast the capabilities of future wide-field-area surveys of galaxy clusters to constrain cosmological parameters. To this aim, we generated a simulated universe obeying the richness-mass scaling that we found. We observed it with a PanStarrs 1+Euclid-like survey, allowing for intrinsic scatter between mass and richness, for errors on mass, on richness, and for photometric redshift errors. We fitted the observations with an evolving five-parameter richness-mass scaling with parameters to be determined. Input parameters were recovered, but only if the cluster mass function and the weak-lensing redshift-dependent selection function were accounted for in the fitting of the mass-richness scaling. This emphasizes the limitations of often adopted simplifying assumptions, such as having a mass-complete redshift-independent sample. We derived the uncertainty and the covariance matrix of the (evolving) richness-mass scaling, which are the input ingredients of cosmological forecasts using cluster counts. We find that the richness-mass scaling parameters can be determined $10^{5}$ times better than estimated in previous works that did not use weak-lensing mass estimates, although we emphasize that this high factor was derived with scaling relations with different parameterizations. The better knowledge of the scaling parameters likely has a strong impact on the relative importance of the different probes used to constrain cosmological parameters. The fitting code used for computing the predicted prior, including the treatment of the mass function and of the weak-lensing selection function, is provided in Appendix A. It can be re-used, for example, to derive the predicted prior of other observable-mass scalings, such as the $L_{\mathrm{X}}$-mass relation.
\end{abstract}

Key words. galaxies: clusters: general - cosmological parameters - cosmology: observations - methods: statistical

\section{Introduction}

If one has a sample of $N$ clusters with measured properties, $o b s n_{i}, z_{i}$ (where $i=1,2, \ldots, N$ ), for example in a Euclid-like survey, their constraints on the cosmological parameters $\theta=$ $\left(\Omega_{\mathrm{M}}, \Omega_{\Lambda}, \sigma_{8}, w, \ldots\right)$ can be derived by applying Bayes's theorem to obtain the posterior distribution of the cosmological parameters,

$p\left(\theta \mid o b s n_{i}, z_{i}\right) \propto p\left(o b s n_{i}, z_{i} \mid \theta\right) p(\theta)$,

where $p(\theta)$ is the prior on cosmological parameters (e.g. from other surveys) and $p\left(o b s n_{i}, z_{i} \mid \theta\right)$ is the likelihood of measuring $N$ clusters with measured properties $o b s n_{i}, z_{i}$. If the mass $M$ were observable, the cosmological parameters $\theta$ would be constrained by fitting $p(M, z \mid \theta)$ to the observed distribution. However, this direct fit is not possible with survey data, because one needs to rely on an observable (mass proxy), such as richness or $Y_{\mathrm{X}}$, and fit the distribution of the observable, $O$ with $p(O, z \mid M)$. To estimate the cosmological parameters, one needs to assume a model for the scaling between the mass and the observable (usually a power law) and some knowledge about how precisely the parameters describing this relation are known (the mass-observable prior): the knowledge may range from very precise (a delta function prior) to very uncertain (e.g. an improper uniform prior).

\footnotetext{
* Appendix $\mathrm{A}$ is available in electronic form at http: //www . aanda.org
}

Of course, cosmological estimates benefit from better known scaling parameters, i.e. priors that enclose a narrow volume of the parameter space that describes the mass-observable scaling.

Most previous forecasts dealing with counts of galaxy clusters (e.g. Lima \& Hu 2005; Sartoris et al. 2010; Carbone et al. 2012) assumed the precision with which the parameters of the mass-observable scaling will be known instead of measuring it. One of the purposes of this work is to quantify this part of the inference step: we aim to compute the uncertainties of the massobservable scaling, i.e. the volume of the mass-richness scaling parameter space enclosed by the posterior probability distribution. We consider, specifically, cluster richness as the mass proxy. This analysis gives us the input prior of cosmological forecasts using cluster counts.

The paper is organized as follow: in Sect. 2 we measure the mass-observable relation in the local Universe from real data, we determine how well the cluster redshift can be inferred from the colour of the red sequence, and we compute in which part of the universe the observable can be measured with current data. In Sect. 3 we assume a fiducial model where the relation between mass and proxy does not evolve. We populate an (simulated) observable universe, we measure the parameter uncertainties by fitting an evolving mass-observable relation to all data (real and simulated), and we test our ability to recover an evolving massobservable relation. Finally, in Sect. 4 we discuss our results and compare the measured uncertainties of the mass-observable 


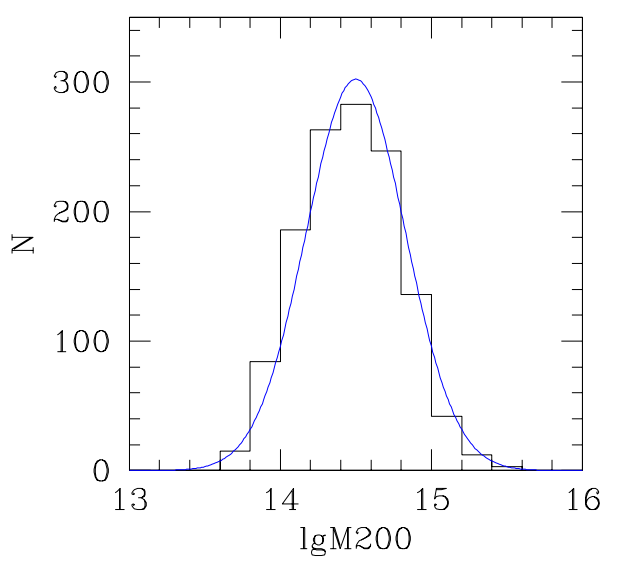

Fig. 1. Computed selection function (histogram) and its adopted Gaussian approximation (curve).

scaling with what has been thus far assumed in cosmological forecasts. Section 5 summarizes the results of this work.

Throughout this paper we assume $\Omega_{\mathrm{M}}=0.3, \Omega_{\Lambda}=0.7$, $H_{0}=70 \mathrm{~km} \mathrm{~s}^{-1} \mathrm{Mpc}^{-1}, \sigma_{8}=0.8$. Magnitudes are quoted in their native system (quasi-AB for SDSS magnitudes). All logarithms in this work are on base ten, unless otherwise indicated. All quantities are measured at the $r_{200 c}$ radius, whose enclosed averaged mass density is 200 times the critical density. The richness-mass calibration in this paper refers to richnesses measured following the Andreon \& Hurn (2010) prescriptions, and therefore cannot be used for other types of richnesses, e.g. Abell (1958) richnesses. We adopt the standard statistical notation: the $\sim$ symbol reads "is drawn from" or "is distributed as" and the $\leftarrow$ symbol reads "take the value of".

\section{Calibration of the mass-proxy from current data}

\subsection{Local calibration of the richness-mass relation based on real data}

In this section, we are interested in the scaling between richness and mass in the local Universe taking into account the noise in their measurement and selection effects.

We re-analysed the very same data that were used in Andreon \& Hurn (2010), adopting the modelling appropriate for the task of current interest. In short, the data consist of cluster richnesses, $n 200$, based on red galaxies measured on specified luminosity and colour ranges within a fiducial radius, and masses derived from the caustic technique computed using 208 galaxies on average per cluster for 53 galaxy clusters at $0.03<z<0.1$. As detailed in Andreon \& Hurn (2010), the parameters describing the mass-richness relation do not change if we use instead velocitydispersion-based masses. We emphasize that we used the values denoted with a hat in Andreon \& Hurn (2010) because they are derived without knowledge of the mass-related quantities $\left(r_{200}\right)$, precisely like in real survey data. For notation simplicity, we here suppress the hat notation adopted there.

Because it is an X-ray selected sample, the considered cluster sample is controlled, not random; therefore, bright clusters are over-represented. In general, a non-random selection causes biases in the recovered regression parameters if the selection is neglected (Gelman et al. 2003; Stanek et al. 2006; Pacaud et al. 2007; Andreon, Trinchieri \& Pizzolato 2011; Andreon \& Moretti 2011; Andreon \& Hurn 2012; and see also Sect. 3.2 where we discuss this problem at length for a sample for which the nonrandom selection cannot be ignored). To be precise, the studied

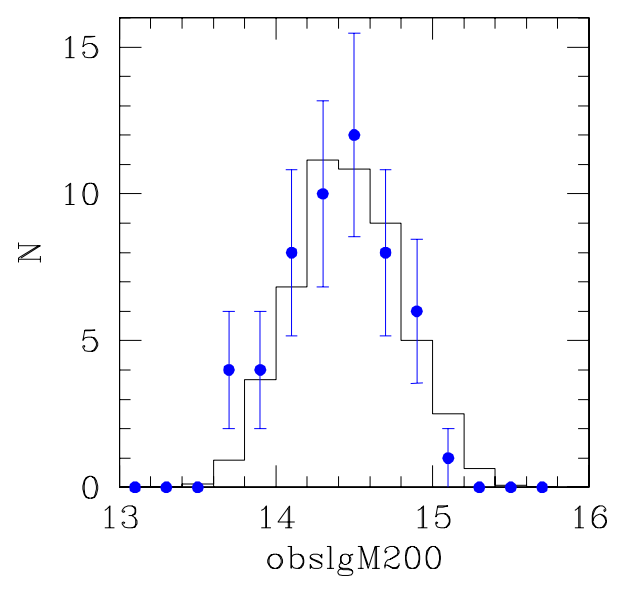

Fig. 2. Distribution of the expected obslgM200 fake data (histogram) and distribution of real data (points). Errorbars mark count standard deviation (i.e. are $\sqrt{n}$ ), not the error.

cluster sample is a random sampling (as detailed in Andreon \& Hurn 2010) of an X-ray selected sample. Its controlled nature allows us to compute the mass selection function, which is essential, in general, to correct for non-random mass selection leading to biases in the recovered regression parameters. We computed the mass selection function (mass prior) as follows: we assumed that the local cluster mass function is described by a Jenkins et al. (2001) mass function at the masses of interest $\left(\log M>13.5 M_{\odot}\right)$. Our results are independent of the chosen parametrization (e.g. if Press \& Schecther 1974, would be adopted). We then followed Stanek et al. (2006): the mean relation between the X-ray luminosity and the mass has a slope equal to 1.59 , intercept equal to $\ln L x_{15}=1.34$ (in a system employing different Hubble constant conventions for luminosity and mass), intrinsic scatter of 0.59 , and the distribution of the (neperian ln) $\mathrm{X}$-ray luminosity at a given mass is Gaussian, i.e.

$$
\ln L_{\mathrm{X}, i} \sim \mathcal{N}\left(1.59\left(\lg M 200_{i}-15\right)+1.34,0.59^{2}\right) .
$$

This ${ }^{1}$ allows us to populate a simulated local universe, $0.03<$ $z<0.1$, with clusters of X-ray luminosity $\ln L_{\mathrm{X}, i}$. The flux of these (simulated) clusters is computed and the objects are kept in the sample if $f_{\mathrm{X}}>3 \times 10^{-12} \mathrm{erg} \mathrm{s}^{-1} \mathrm{~cm}^{-2}$, which is the flux threshold adopted by Rines \& Diaferio (2006), the parent sample from which Andreon \& Hurn (2010) studied a random subsample. Figure 1 shows the result of this simulation, and the adopted analytic (Gaussian) parametrization:

$\lg M 200 \sim \mathcal{N}\left(14.5,0.33^{2}\right)$.

Assuming Eq. (3), we computed the expected distribution of the observed values of $\lg$ M200, obslgM200 of our simulated survey, assuming a common error for the mass error, 0.14 dex, the average value of the studied sample. We compared this to the actual observed distribution (i.e. real data) in Fig. 2. The agreement is impressive (there are no free parameters to tune), showing that our modelling of the selection function captures the data behaviour and gives us $p(\lg M 200)$ i.e. the probability that a cluster has mass $\lg M 200$ and is included in the sample (i.e. the mass prior). The derived $p(\lg$ M200) allows us to avoid

\footnotetext{
1 The tilde symbol indicates a similarity subject to stochasticity, either because of noise or because of intrinsic differences among members. In other words, the tilde symbol indicates that we account for uncertainty or non-homogeneity (variety).
} 
the biases coming from the non-random mass distribution of our sample.

We proceed by specifying the assumed mathematical dependence between the quantities involved in our problem. We need to acknowledge the uncertainty in all measurements and therefore, because of errors, observed and true values are not identically equal. The variables $n 200_{i}$ and $n b k g_{i}$ represent the true richness and the true background galaxy counts in the studied solid angles. We measured the number of galaxies in both cluster and control field regions, obstot $_{i}$ and $o b s b k g_{i}$ respectively, for each of our 53 clusters (i.e. for $i=1, \ldots, 53$ ). We allowed Poisson errors for both and we assumed that all measurements are conditionally independent. The ratio between the cluster and control field solid angles, $C_{i}$, is known exactly. In formulae:

$$
\begin{aligned}
& \text { obsbkg }_{i} \sim \mathcal{P}\left(n b k g_{i}\right) \\
& \text { obstot }_{i} \sim \mathcal{P}\left(n b k g_{i} / C_{i}+n 200_{i}\right) .
\end{aligned}
$$

For each cluster, we have a cluster mass measurement and a measurement of the error associated with this mass, obslgM200 and obserrlg $M 200_{i}$ respectively. We allowed Gaussian errors on mass:

$$
\text { obslgM200 }_{i} \sim \mathcal{N}\left(\lg M 200_{i}, \text { obserrlgM200 }_{i}^{2}\right) .
$$

We assume a power law relation between mass and $n 200$ with intercept $\alpha+1.5$, slope $\beta$ and intrinsic scatter $\sigma_{\text {scat }}$ :

$$
\operatorname{lgn} 200_{i} \sim \mathcal{N}\left(\alpha+1.5+\beta\left(\log \left(M 200_{i}\right)-14.5\right), \sigma_{\text {scat }}^{2}\right) .
$$

The quantity $\log (M 200)$ is centred at an average value of 14.5 and $\alpha$ is centred at 1.5 , for computational advantages in the MCMC algorithm used to fit the model (it speeds up convergence, improves chain mixing, etc.) and to reduce the covariance between parameters. The relation is between true values, not between observed values, which may be biased.

The priors on the slope and the intercept of the regression line in Eq. (7) were taken to be quite flat, a zero mean Gaussian with very strong variance for $\alpha$ and a Students- $t$ distribution with one degree of freedom for $\beta$. The latter choice was made to avoid that properties of galaxy clusters depend on astronomer rules of measuring angles (from the $x$ or from the $y$ axis). This agrees with the model choices in Andreon (2006 and later works). Our $t$ distribution on $\beta$ is mathematically equivalent to a uniform prior on the angle $b$. In formulae:

$\alpha \sim \mathcal{N}\left(0.0,10^{4}\right)$

$\beta \sim t_{1}$.

For the true values of the background, we chose to impose no strong a-priori values, only enforcing positivity, by adopting an improper uniform prior,

$n b k g_{i} \sim \mathcal{U}(0, \infty)$

Fitting our sample of 53 clusters with the model above, we found

$$
\operatorname{lgn} 200=(0.47 \pm 0.12)(\lg M 200-14.5)+1.58 \pm 0.04 .
$$

Unless otherwise stated, the results of the statistical computations are quoted in the form $x \pm y$ where $x$ is the posterior mean and $y$ is the posterior standard deviation. All statistical computations were performed using JAGS (Plummer 2010), see Appendix A for an example.



Fig. 3. Richness-mass scaling. The solid line marks the mean fitted regression line of $\log (n 200)$ on $\lg M 200$, while the dashed lines show this mean plus or minus the intrinsic scatter $\sigma_{\text {scat }}$. The shaded region marks the $68 \%$ highest posterior interval for the regression. Error bars on the data points represent observed errors for both variables. The distances between the data and the regression line is due in part to the measurement error and in part to the intrinsic scatter.

Figure 3 shows the scaling between richness and mass, the observed data, the mean scaling (solid line), and its $68 \%$ uncertainty (shaded yellow region), and the mean intrinsic scatter (dashed lines) around the mean relation. The \pm 1 intrinsic scatter band contains $60 \%$ of the data points and is not expected to contain $68 \%$ of them, because of the measurement errors.

Figure 4 shows the posterior marginals for the key parameters, i.e. for the intercept, slope, and intrinsic scatter $\sigma_{\text {scat }}$. These marginals are reasonably well approximated by Gaussians. The intrinsic mass scatter at a given richness, $\sigma_{\text {scat }}=\sigma_{\lg M 200 \mid \log n 200}$, is small, $0.25 \pm 0.03$ dex. These posterior probability distributions are dominated by the data (their widths are much smaller than the prior widths), i.e. our results are independent of the assumed prior to all practical effects. Parameters show no appreciable covariance (figure not shown) because of our choice of zero-pointing masses near the data average (Eq. (7)). This allows a simpler summary of the posterior, which we use in our next inference step (Eqs. (17) to (19)).

We note that these results are almost indistinguishable from results we might obtain without modelling the selection function, basically because the prior is broad compared to $\lg M 200$ errors.

\subsubsection{Side comments}

Cosmological forecasts dealing with cluster counts in the optical sometimes use the scatter between observable and mass from Rykoff et al. (2012) or Rozo et al. (2009). It is worth emphasizing that to measure the scatter between two quantities, it is strongly preferable to have both. Neither of these two works have individual values of cluster masses.

It is worth remembering that the slope of the direct relation is not the inverse of the slope of the inverse relation, i.e. if $O \propto M^{\gamma}$, then usually $M \not k O^{1 / \gamma}$ (e.g. Isobe et al. 1990; Andreon \& Hurn 2010). Therefore, it is not surprising that the slope between mass and richness is not the reciprocal of the slope determined in Andreon \& Hurn (2010) for the inverse relation using the very 

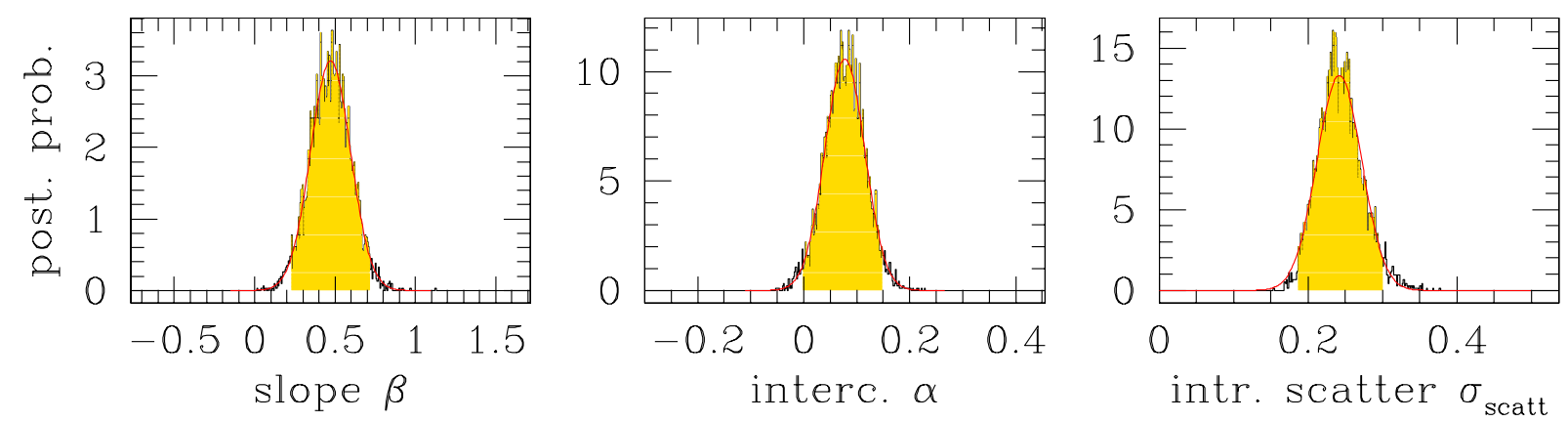

Fig. 4. Posterior probability distribution for the parameters of the richness-mass scaling computed from the real data. The black jagged histogram shows the posterior as computed by MCMC, marginalised over the other parameters. The red curve is a Gaussian approximation of it. The shaded (yellow) range shows the $95 \%$ highest posterior credible interval.

same data. Furthermore, the slope depicted in Fig. 3 is not "too shallow" compared to the data, a steeper slope would systematically over- or underestimate the cluster richness (see Andreon \& Hurn 2010, 2012 for a brief astronomical introduction on regression fitting).

\subsection{In which part of the Universe is richness measurable with current data?}

The cluster richness was derived using $g-r$ colour and luminosities of galaxies brighter than an evolving limiting magnitude $M_{V}^{\mathrm{e}}<-20$.

Figure 5 illustrates how depth and colour constraints change with redshift. The top panel illustrates the apparent luminosity of a red $M_{V}^{\mathrm{e}}=-20$ mag galaxy, modelled as a $z_{f}=5$ single stellar population using the 2007 version of the Bruzual \& Charlot (2003) synthesis population model for different filters: $g, r, i$, and $z$ for the $3 \pi$ Steradian PanStarrs 1 survey (PS1, hereafter) and riz, $Y, J$, and $H$ for Euclid ${ }^{2}$ (Laureijs et al. 2011) with the corresponding $\sim 10 \sigma$ depth (horizontal ticks). For the $3 \pi$ PanStarrs 1, we took the current depth, i.e. that already achieved after the first two years of observation (Kaiser, priv. comm.). The PS1 has a Y-like filter, not plotted because it is shallower than the Euclid Y. The Dark Energy Survey (Abbott et al. 2005) is deeper than PS1, but covers a smaller solid angle. The Euclid consortium plan to have ground based griz data deeper than our need over the whole $15000 \mathrm{deg}^{2}$ survey area (Laureijs et al. 2011).

The bottom panel illustrates the wavelength range sampled by these filters. Only redshift bins where galaxies are brighter than the $10 \sigma$ depth are plotted. The shaded yellow is the $\lambda$ range sampled by $g-r$ at $z<0.08$. As the figure shows, we always have at least two filters in the shaded region, i.e. up to $z=1$ at least these data have appropriate depth and wavelength coverage to count galaxies. Indeed, the $M_{V}^{\mathrm{e}}<-20$ mag cut was chosen to precisely perform this measurement on ten-year old MOSAIC-II CTIO images up to $z=0.82$ (e.g. those in Andreon et al. 2004a). These depths are routinely achieved in current surveys, such as the CFHTLS (Cuillandre \& Bertin 2006).

To summarize, incoming (and also current) surveys have the depth and filter coverage adequate to compute the number of red galaxies needed to derive $n 200$. Furthermore, Andreon (2012) showed that the galaxy background $\left(\mathrm{nbkg}_{i} / C_{i}\right.$ in Eq. (5)) is negligible even at magnitudes fainter than those adopted in this work, and not detrimental at all for the derivation of the cluster richness.

\footnotetext{
2 http://www.euclid-ec.org
}



Fig. 5. Depth and wavelength coverage of the two-year PS1 and Euclid surveys. Upper panel: $g, r, i$, and $z$ (from left to right) filters are indicated with dashed (blue, green, red, and black) lines. riz, $y, J$, and $H$ (from left to right) Euclid filters are indicated with thick solid (blue, green, red, and black) lines. The horizontal tick indicates the $\sim 10 \sigma$ depth, most of them are at $z>1$ and thus not visible in the plot. Bottom panel: wavelength coverage of the filters for redshift bins where galaxies are brighter than the $\sim 10 \sigma$ depth. The shaded (yellow) region marks the wavelength sampling of $g-r$ at $z \sim 0$.

\subsection{Which precision for photometric redshift?}

Surveys such as those performed by PanStarrs 1, DES, or Euclid will detect thousands of clusters and it is unreasonable to expect that all of them will have a spectroscopic redshift. How precise will their redshift estimate be? We can set a conservative estimate by considering current shallower surveys that sample similar redshifts.

We considered spectroscopic and photometric redshifts of the sample of 228 clusters at $0.06<z<0.3$ in Andreon (2003a,b) and the $160.3<z<0.9$ clusters in Andreon et al. (2004a, 2004b). They are all colour-detected with the redsequence method of Andreon (2003a), which is an adaptation 


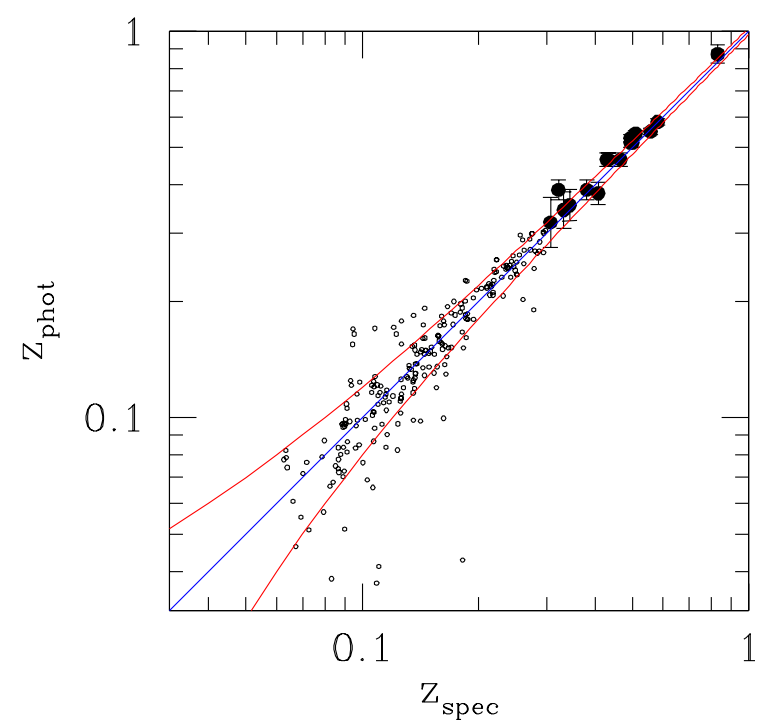

Fig. 6. Red sequence photometric redshift performance. Spectroscopic redshift vs. photometric redshift from the colour of the red sequence in $g-r$ (small open points, error bars are not plotted to avoid crowding, there are 228 plotted points, sometime one on top of the other) or $R-z^{\prime}$ (solid points with error bars). The $z_{\text {phot }}=z_{\text {spec }}$ line and the $z_{\text {phot }}=z_{\text {spec }} \pm$ 0.02 loci are indicated with solid lines.

of the Gladders \& Yee (2000) original method (see Andreon 2003a for details) in either the SDSS early-data release area or in the XMM-LSS field. These clusters tend to be of low richness and therefore to have a less prominent red sequence than that of the massive clusters that we consider below. For both samples, the colour of the red sequence was determined using two-band photometry only, $g-r$ (at $z<0.3$ ) or $R-z^{\prime}$ (at $z>0.3$ ). The photometric redshift was derived from the colour of the red sequence adopting a relation between redshift and colour (an empirical template at $z<0.3$, an old galaxy template at higher redshift, as detailed in Andreon 2003a and Andreon et al. 2004a, respectively). Figure 6 shows $z_{\text {phot }}$ vs. $z_{\text {spec }}$ for the 244 clusters, the (straight line) $z_{\text {phot }}=z_{\text {spec }}$ line and the $z_{\text {phot }}=z_{\text {spec }} \pm 0.02$ loci. Twenty-five percent of the points have $\left|z_{\text {phot }}-z_{\text {spec }}\right|>0.02$, while $>32 \%$ are expected if the photometric error is 0.02 . Even restricting the attention to $z>0.3,6$ clusters show $\left|z_{\text {phot }}-z_{\text {spec }}\right|>\sqrt{0.02^{2}+e r r_{\text {phot }}^{2}}$ vs. 5.1 expected cases if the redshift derived from the red sequence has an intrinsic scatter of 0.02 . This implies that we can already achieve a $\Delta z=0.02$ precision using the colour of the red sequence using two bands. Similar results were found by Puddu et al. (2001) for a small, but X-ray selected (and therefore more massive) cluster sample, and by High et al. (2010) for a small, but mostly at $z>0.3$, cluster sample. In both cases the estimate of the clusters' redshift is based on the colour of the red sequence.

The extremely good performance of the red sequence colour as a redshift indicator is hardly surprising because of the implicit selection of one single type of galaxies with a distinctive $4000 \AA$ break (spectrophotometric bright early-type galaxies) and of the colour homogeneity of the early-type galaxy class (e.g. Stanford et al. 1998; Kodama et al. 1998; Andreon 2003a,b; Andreon et al. 2004a).

In summary, we can safely assume for future clusters a (conservative) 0.02 error on cluster (photometric) redshifts, because this performance is already achieved today using the colour of the red sequence.

\section{Calibration with future surveys}

\subsection{Generation of mock-calibration Euclid data}

We generated a Monte-Carlo simulated universe obeying to the mass-richness scaling we just computed and observed it with a PanStarrs 1+Euclid-like survey. Our fiducial universe has unevolving parameters that describe the mass-richness scaling. A Euclid-like survey is needed to measure cluster masses, whereas for the computation of cluster richness one needs shallower, but multicolour data, such as already acquired by the PanStarrs 1 survey.

We followed Bergé et al. (2010) to compute the number (the probability times the volume) of clusters in the Euclid-wide survey at redshift $z$, with mass $\lg M 200$, which produces a weaklensing signal with a given signal-to-noise ratio $(\mathrm{S} / \mathrm{N})$. We used the halo model with an NFW (Navarro et al. 1997) profile, a Jenkins (2001) mass function, and a modified Sheth et al. (2001) bias (see the Bergé et al. 2010, Appendix for a detailed description). We assumed a galaxy shape noise $\sigma_{\text {int }}=0.3$, and a galaxy number density $n_{\mathrm{g}}=30 \mathrm{arcmin}^{-2}$. We also assumed that all halos are spherical and therefore did not account for the shape bias described by Hamana et al. (2012). Projection effects are, in these observational conditions and for clusters as massive as those of interest in our paper, largely sub-dominant (Marian \& Bernstein 2006), and were neglected for this reason. For the Euclid survey, we adopted the updated sky coverage $\left(15000 \mathrm{deg}^{2}\right)$. The iso-density contours in Fig. 6 indicate lines where we expect 1,10 , and 100 clusters with an $S / N>5$ per bin of 0.1 dex in mass and 0.0275 in redshift in the Euclid survey. The minimal $S / N=5$ mass, $\lg$ M200trunc, is well described by

$\lg$ M200truc $=13.9891+1.04936 z+0.488881 z^{2}$.

We exploited these masses to calibrate the richness-mass relation and its evolution.

First of all, we generated a Monte-Carlo realization of the Bergé et al. (2010) distributions. Then, we selected $S / N>5$ detections only, because we did not want to deal with too noisy measurements (Hamana et al. 2012; Pace et al. 2007). Furthermore, we removed clusters at $z<0.03$ to avoid very nearby clusters with bright and large galaxies whose photometry will likely be corrupted ${ }^{3}$. This left us about 11000 clusters with available $z_{i}, \lg M 200_{i}$, and $(S / N)_{i}$.

The cluster masses were then observed, i.e. mass errors were taken to be Gaussian and equal to errlgM200 $i=\frac{1}{S / N} / \ln (10)$, where the latter term is due to our choice of measuring errors using decimal logarithms:

obslgM200 $_{i} \sim \mathcal{N}\left(\lg M 200_{i}\right.$, errlgM $\left._{200}^{2}\right)$.

Cluster richnesses were assigned to simulated clusters assuming the model measured in the local Universe, i.e. (Sect. 2.1)

$\operatorname{lgn} 200_{i} \sim \mathcal{N}\left(0.47\left(\lg M 200_{i}-14.5\right)+1.58,0.25^{2}\right)$.

We emphasize, once more, that we allowed for an intrinsic scatter, i.e. we allowed clusters of a given mass to have a variety of richnesses. Richnesses were then observed: richness, as all measurements in this paper, have errors, which were assumed to be Poissonian,

obslgn200 $\sim \mathcal{P}\left(\operatorname{lgn} 200_{i}\right)$.

3 For example in the SDSS, which is much shallower and therefore less tailored for faint galaxies, photometry of galaxies at $z<0.02$ suffers from shredding problems. 


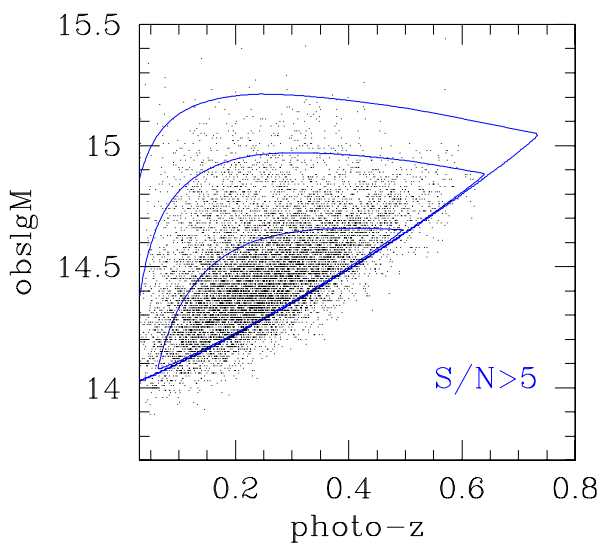

Fig. 7. Contours: number of clusters for which weak-lensing mass estimates can be obtained by a Euclid-like survey. From outer to inner contours the lines represent isocontours of $S / N>5$ weak-lensing detection of 1,10 , and 100 clusters as a function of redshift and mass. Points: a Poisson realization of the above, with errors on mass and redshift (these move points outside the $N=1$ contour).

Finally, we also allowed Gaussian photometric errors, taken to be 0.02 at all redshifts (see Sect. 2.3):

$o b s z_{i} \sim \mathcal{N}\left(z, 0.02^{2}\right)$.

This procedure yielded 10714 clusters with measured obslgn $200_{i}$, obslgM200 ${ }_{i}$, errlgM200 ${ }_{i}$, and $o b s z_{i}$, that we used to determine the relation between richness and mass and its evolution. Figure 7 depicts them individually (points). Because of mass errors, there are points below the minimal $S / N=5$ mass (the diagonal slightly bent line). The average richness (obsn200) of the simulated sample is 46 galaxies, while the median is 38 galaxies. Two thirds of them are at $z<0.3$, where the scatter between redshift and photometric redshift is better sampled by our real data (Sect. 2.3). The generated sample does not contain any cluster with a weak-lensing detection at $S / N>5$ at $z>0.62$ (Fig. 7).

\subsection{Determining the richness-mass predicted priors}

We now combined the real data from the local Universe with the simulated data (depicted in Fig. 7), to compute how well we are able to measure the richness-mass scaling at all redshifts. In this section we will not use true values because these are unknown for the real data. Furthermore, we cannot assume to know how the parameters of the richness-mass scaling evolve, because this is precisely what we want to infer from the data.

The information encoded in the local Universe (Sect. 2.1) is the current prior:

$$
\begin{aligned}
\sigma_{\text {intrscat }} & \sim \mathcal{N}\left(0.25,0.03^{2}\right) \\
\alpha & \sim \mathcal{N}\left(0.08,0.04^{2}\right) \\
\beta & \sim \mathcal{N}\left(0.47,0.12^{2}\right) .
\end{aligned}
$$

We assumed that the scatter and the intercept may both change with redshift:

$$
\begin{aligned}
\operatorname{lgn} 200 m_{i} & \leftarrow \alpha+1.5+\beta\left(\lg M 200_{i}-14.5\right)+\gamma \ln \left(1+z_{i}\right) \\
\operatorname{lgn} 200_{i} & \sim \mathcal{N}\left(\operatorname{lgn} 200 m_{i}, \sigma_{\text {intrscat }}^{2}\left(z_{i}\right)\right) \\
\sigma_{\text {intrscat }}^{2}\left(z_{i}\right) & \leftarrow \sigma_{\text {intrscat }}^{2}-1+\left(1+z_{i}\right)^{2 \zeta}
\end{aligned}
$$

While the adopted modelling of the evolution is common in previous works (e.g. Sartoris et al. 2010; Carbone et al. 2012), we emphasize that a different modelling is possible and legitimate. We also emphasize that, as in previous works, we assumed to perfectly known the analytic expression of the distribution function of the intrinsic scatter term (a Gaussian), when its shape should be left more flexible, or at very least, checked with data, because this uncertainty may be dominant (Shaw et al. 2010). Equation (21) and the fitting code (given in Appendix A) may be easily modified replacing the adopted Gaussian with a more flexible distribution, e.g. by a mixture of two Gaussians, which guarantee a valid (positive) probability distribution, unlike the Edgeworth series expansion proposed in Shaw et al. (2010).

We adopted weak priors for the newly introduced parameters: as prior for the $\gamma$ and $\zeta$ slopes we adopted a Students $t$ distribution centred on zero with one degree of freedom, as for the slope $\beta$ in Sect. 2.1, to make our choice independent of astronomer rules of measuring angles. In formulae:

$\gamma \sim t_{1}$

$\zeta \sim t_{1}$.

As in previous sections, richness has Poisson errors:

obsn200 $i \sim \mathcal{P}\left(n 200_{i}\right)$,

whereas masses and photometric redshifts have Gaussian errors:

$$
\begin{aligned}
\operatorname{obslgM200}_{i} & \sim \mathcal{N}\left(\lg M 200_{i}, \text { errlgM200 }_{i}^{2}\right) \\
o b s z_{i} & \sim \mathcal{N}\left(z, 0.02^{2}\right) .
\end{aligned}
$$

To complete the model description, we need to specify the mass prior. We cannot ignore that the mass function is steep and that the weak-lensing $S / N>5$ cut introduces an abrupt discontinuity: ignoring them would lead to a biased fit (the recovered slope would be much shallower than the input one) due to a Malmquist-like bias. Indeed, mass errors tend to make the distribution in mass broader, especially at low-mass values, because of the sharp $S / N=5$ weak-lensing detection requirement, but also at high-mass values because of the steepness of the mass function. Since high-mass values are overestimated and low-mass values are underestimated, any quantity that is fitted against these (biased) values neglecting the selection function would return a shallower relation (see also Andreon \& Hurn 2010, for the similarly biased mass-richness relation of Johnston et al. 2007). For mathematical simplicity and given the small mass range explored, we modelled the Jenkins et al. (2001) mass distribution at a given redshift as a Schechter (1976) function with slope -1 and characteristic mass given by

$\lg M 200^{*}=12.6-(z-0.3)$

truncated at lgM200truc, given by Eq. (12). The parameters of Eq. (28) were determined by fitting the Jenkins et al. (2001) mass function.

On the other hand, we do not need to model the optical cluster selection function, because the large cluster richness and the photometric depth allow all clusters that produce a detectable weak-lensing signal to be easily detectable as overdensities of red galaxy because they have, on average, 38 galaxies projected on a background of (nearly) zero galaxies.

We do not need, either, to accurately model the redshift prior, because photometric redshifts are well-determined. We can therefore assume an uniform distribution for it

$z_{i} \sim \mathcal{U}(0,1)$ 



Fig. 8. Marginal (panels on the diagonal) and joint (other panels) probability distributions of the mass-richness scaling derived from real and simulated data for a PS1+Euclid-like survey. Red jagged contours and histograms refer to probabilities computed from the MCMC sampling, while the blue smooth contours/lines refer to a Gaussian approximation of the numerically found posterior. Contours are at $68 \%$ probability level. Vertical (cyan) lines and crosses indicate the values used to generate the data, while the dashed (green) lines show the current low-redshift calibration of the richness-mass scaling.

although we emphasize that for large photometric redshift errors one should account for gradients in $n(z)$.

We emphasize that modelling the mass- and selection function is compulsory; not accounting for it would lead to a fitted slope $\gg 5 \sigma$ different from the input one. Therefore, results based on methods that do not allow one to account for the mass- and selection function, e.g. the usual linear regression analysis based on BCES (Akritas \& Bershady 1996), or simplistic forecast analyses lacking any treatment of the selection function (as is typical of Fisher analyses), should be used with great caution. On the other hand, one should not be overly anxious about modelling the mass- and selection function: what matters is their general shape, which drives the correction of the bias, not their precise shape, i.e. whether the mass function is a Tinker et al. (2008) or Jenkins et al. (2001) mass function, for instance. The uncertainty on the precise shape of the mass function, neglected in this work because of the small considered mass range, is an uncertainty of secondary importance compared to the large uncertainy involved through the mass errors. The main point to keep in mind is that the mass function is certainly not uniform, it is evolving with redshift, the clusters entering in the sample are not a random sampling of the mass function (all those with low mass are excluded, and the limiting mass is changing with redshift) and we account for that (not accounting leads to parameters off by $\gg 5 \sigma$, as mentioned), while other observable-mass fitting models (sometime implicitely) assume a uniform prior on cluster mass and mass-random selection, unless differently specified.

The software implementation of this fitting model is given in Appendix A.

Fitting the simulated+real data with this model returns parameters whose (posterior) probability distributions are depicted in Fig. 8. Figure 8 and its summary in Table 1 are one of the main results of this work, since they are the priors (starting points) needed to forecast cosmological parameters with cluster data.

Marginal probabilities are shown on the diagonal, while the other panels show the joint probability distributions, i.e. the covariance between pairs of parameters. Each panel reports two closely packed lines: the red one is the Laplace (Gaussian) approximation of the posterior, while the histogram/jagged contour is the straight outcome of the numerical computation (somewhat 
Table 1. Predicted richness-mass prior parameters for a PS1+Euclidlike survey: covariance matrix $\sigma_{i, j}$.

\begin{tabular}{cccccc}
\hline \hline & $\alpha$ & $\beta$ & $\sigma$ & $\gamma$ & $\zeta$ \\
$\alpha$ & $5.9 \times 10^{-5}$ & & & & \\
$\beta$ & $5.4 \times 10^{-5}$ & $2.1 \times 10^{-4}$ & & & \\
$\sigma$ & $-2.1 \times 10^{-6}$ & $5.9 \times 10^{-7}$ & $2.6 \times 10^{-5}$ & & \\
$\gamma$ & $-2.0 \times 10^{-4}$ & $-2.4 \times 10^{-4}$ & $7.5 \times 10^{-6}$ & $1.0 \times 10^{-3}$ & \\
$\zeta$ & $1.7 \times 10^{-6}$ & $-1.8 \times 10^{-7}$ & $-2.5 \times 10^{-5}$ & $-7.2 \times 10^{-6}$ & $2.9 \times 10^{-5}$ \\
\hline
\end{tabular}

noisy because of the finite length of the MCMC chain). The Laplace (Gaussian) approximation captures the probability distributions well.

The diagonal panels also show the input values (vertical lines). They are all within 1.5 posterior standard deviations from the recovered value ${ }^{4}$. By fitting the observed data, we recover the five parameters that describe the mass-richness scaling with good accuracy and without bias.

In addition to input values, the diagonal panels show the current low-redshift calibration of the richness-mass scaling (dashed green line). Euclid masses significantly improve the current low-redshift calibration of the richness-mass scaling: the intercept $\alpha$, currently known to within $10 \%$ (0.04 dex, Sect. 2.1), will be known with a per cent accuracy, the slope $\beta$, currently known with a sizeable uncertainty $(0.47 \pm 0.12$, Sect. 2.1$)$ will have its uncertainty reduced by a factor 10 . The intrinsic scatter, currently known with a $\sim 10 \%$ accuracy (Sect. 2.1 ), will be known with a per cent accuracy. The evolution of the intrinsic scatter and of the intercept will be known with a 0.03 and 0.005 uncertainty, respectively. The computed posterior is $\sim 10^{3}$ times narrower (in the $\alpha-\beta-\sigma$ space) than the current calibration of the richness-mass scaling, a significant improvement over the current low-redshift calibration. This capability makes the Euclid mission unique and independent of the success of observations other than the already acquired PanStarrs 1 multicolour data. Instead, the calibration of the mass-proxy relation of the XXLS cluster survey (Pierre et al. 2011) must rely on the success of an expensive XMM calibration program (Pierre et al. 2011), which is not yet implemented. Similarly, the SPT survey requires an external calibration. Although the current clusters sample consists only of 100 clusters (Reichardt et al. 2012), the currently available calibration, not the sample size, is the main source of uncertainty in cosmological estimates.

There is a strong covariance between the evolution and the $z=0$ value of the intercept ( $\gamma-\alpha$ panel of Fig. 8). It can be easily understood by noting that $z=0$ is outside the range of sampled redshifts. The covariance between intrinsic scatter and its evolution ( $\zeta-\sigma_{\text {intrscat }}$ panel of Fig. 8) has a similar origin: the intrinsic scatter is defined at an un-observed redshift, $z=0$, instead of a redshift where it is well observed.

Figure 9 compares the model fit (solid line) to the true input relation in stacks of 201 clusters per point. The model fit on noisy data and the (unobserved and unused in the analysis) noise-less data agree well, indicating that the fit to the noisy data captures the real trend of the noise-less true data well.

In summary, by fitting observed data we recover with good accuracy and without bias the five parameters describing the mass-richness scaling. In particular, we assumed no evolution (i.e. $\gamma=0$ and $\zeta=0$ ) and recovered it, even allowing evolution on both scatter and intercept. We will be able to measure the

\footnotetext{
4 There is only a $10 \%$ probability that in a five parameter fit all fitted values are found within $1 \sigma$ from the input values, and a $50 \%$ probability that they are all within $1.5 \sigma$.
}



Fig. 9. Richness-mass scaling for the simulated PS1+Euclid-like data. The solid line marks the regression line fitted on observed data. The shaded region marks the $68 \%$ highest posterior credible interval for the regression. The red dashed line indicates the input relation. The data points are stacks of true data in bins of 201 clusters each, true data were never used in the fitting.

mass-richness scaling with an error (posterior parameter standard deviation) of $0.007,0.014,0.005,0.033$, and 0.005 in $\alpha, \beta$, intrinsic scatter, $\gamma$, and $\zeta$, respectively. These are the predicted prior widths of cosmological forecasts. Table 1 lists the covariance matrix.

Strictly speaking, conclusions of this sub-section only hold if our modelling of the richness-mass scaling is a reasonable approximation of the scaling in the real Universe. Therefore it does not hold if, for example, the richness-mass scaling suddenly disappears in the real Universe at $z=0.3$, for instance.

\subsection{What happens if $\sigma_{\text {intrscat }}$ doubles by $z=0.6$ ?}

To understand how the predicted prior is sensitive to a possible evolving mass-proxy scaling, we generated new data with $\zeta=0.18$, i.e. generated from a relation whose intrinsic scatter is twice as large at $z=0.6$ as at $z=0$. To this aim, we replaced equation 14 by

$$
\begin{aligned}
\operatorname{lgn} 200_{i} & \sim \mathcal{N}\left(0.47\left(\lg M 200_{i}-14.5\right)+1.58, \sigma_{\text {intrscat }}^{2}\left(z_{i}\right)\right) \\
\sigma_{\text {intrscat }}^{2}\left(z_{i}\right) & \leftarrow 0.25^{2}-1+\left(1+z_{i}\right)^{2 \zeta} \\
\zeta & \leftarrow 0.18
\end{aligned}
$$

and re-generated the new (simulated) data. We fitted real+simulated data with no change whatsoever, and, as for a non-evolving intrinsic scatter, we recovered the input parameters, finding $\zeta=0.16 \pm 0.01$ (vs. input $\zeta=0.18$ ). The other four parameters were all recovered to better than their uncertainty. More precisely, we found an error of $0.01,0.02$, $0.008,0.046$, and 0.010 in $\alpha, \beta$, intrinsic scatter, $\gamma$, and $\zeta$, respectively. These are larger (1.5 times, on average) than before because with the larger scatter (at high redshift) more data are needed to measure the mean relation with the same precision. Nevertheless, the parameter volume they encompass is only a factor nine larger than for a non-evolving intrinsic scatter, a negligible factor (a mere 0.01 per cent) compared to what 
we discuss below. Marginal and joint probability distributions (i.e. the covariance matrix and Fig 8 revised for these data) are qualitatively similar, apart from the obvious $\approx 1.5$ factor.

In summary, an increasing intrinsic scatter, if present, would be easily recovered from the data, with only a mild degradation of the overall performances (a factor 9 for a five dimensional volume), and no bias.

\section{Discussion}

Our computation of the predicted prior of the mass-richness scaling while not accounting for sub-dominant sources of error, such as uncertainties related to projection and redshift-dependent errors on the cluster photometric redshift, can easily take them into account, it is just a matter of replacing the assumed likelihoods distributions (Eqs. (25) to (27)) with the updated distributions accounting for additional error terms one may wish to consider. For example, we can change the normal intrinsic scatter (questioned by Shaw et al. 2010) into a Student- $t$ distribution by typing less than ten characters (see Appendix for details). However, more complex simulated data (e.g. based on an $N$-body simulation) are needed to generate the data to be fitted and more and better real data are needed to characterize the real additional dependencies (e.g. how to model the intrinsic scatter).

The starting point of literature forecasts is the end point of this paper: they assume what our paper computes, their prior widths are our posterior parameter uncertainties. The predicted prior is computable and thus does not need to be assumed. Parameters show covariance, sometimes a strong one, while none is assumed in literature forecasts (that we are aware of).

We note that the previous literature (starting perhaps with Lima \& Hu 2005) chose not to model the slope between mass and proxy, i.e. implicitly assumed to know it perfectly. This assumption seems optimistic because the slope is presently known with $25 \%$ accuracy (Sect. 2.1, summarized in Eq. (19)). Section 2.3 shows that it will be known after PS1+Euclid with a per cent accuracy. If a perfect knowledge of the slope is assumed, then uncertainties on the other scaling parameters (scatter, intercept, and their evolution) will be underestimated. Furthermore, while the quality of a mass proxy is lower at the ends of the calibration range because of the slope uncertainty, the choice performed in previous literature works makes it a constant quality at all masses, including those outside the range of the calibration sample.

As mentioned above, most previous works (e.g. Lima \& Hu 2005; Cunha \& Evrard 2010; Thomas \& Contaldi 2011; Carbone et al. 2012, etc.) adopted priors for the mass-observable scaling largely by guessing how well the relation is (or will be) known, instead of computing the prior width. Sometimes, the prior width on some key parameters, like the scatter, was taken to be zero. Some works (e.g. Cunha \& Evrard 2010; Oguri \& Takada 2011) explored the sensitivity of cosmological constraints on the adopted priors for the mass-observable scaling, sometimes calling this sensitivity "systematics", quantifying the (obvious) fact that poorly calibrated scaling relations give poor cosmological constraints. For example, Cunha \& Evrard (2010) showed that cosmological constraints easily deteriorate by a factor from $\sqrt{2}$ to 2 by changing the prior width from zero to $\sim 1 \%$.

Even more important, previous forecasts did not use the information content in the weak-lensing masses to calibrate the mass-observable scaling. For comparison, we consider the priors assumed in Carbone et al. (2012), who also considered the mass-richness scaling of a Euclid-like survey, but made no use of the Euclid weak-lensing masses to calibrate the mass-richness scaling. Before proceeding in this comparison, we emphasize a technical difference: the two modellings are identical after swapping observable and mass variables. For example, we modelled the scatter in proxy at a given mass as Gaussian, while Carbone et al. (2012) modelled the scatter in mass at a given proxy as Gaussian. Since the Carbone et al. (2012) model has no slope parameter, for the purpose of this comparison only, we removed the slope from the modelling (freezing it at the true value).

Figure 10 compares the prior adopted in Carbone et al. (we emphasize once more the variable swapping) with our predicted prior. A major point emerges: the parameter volume encompassed by the Carbone et al. prior, which does not use weaklensing to calibrate the mass-richness scaling, is $10^{5}$ times larger (in the $\alpha-\sigma-\gamma-\zeta$ space) than the one we derive using Euclid weak-lensing masses. Similarly, the Euclid imaging consortium science book (EICSB, Refregier et al. 2010) does not use the Euclid weak-lensing masses to calibrate the mass-richness scaling and assumes a $25 \%$, or 0.25 , prior uncertainty on each parameter of an observable-mass relation modelled with a third, different, parametrization. At face value, given that our precisions are typically one order of magnitude better per parameter, using weak-lensing masses may allow us to improve the knowledge of the observable-mass scaling by a similarly large $\left(\approx 10^{5}\right)$ amount. If the mass-proxy scaling can be computed $10^{5}$ times better, stronger cosmological constraints can probably be inferred and this may alter the balance between the cosmological constraints achievable using cluster counts, BAOs, SNae, and weak-lensing tomography. Indeed, Carbone et al. (2012) estimated that if the regression parameters were perfectly known, then cosmological constraints tighten (technically: the volume of cosmological parameter space enclosed by the posterior probability distribution decreases) by a factor $\approx 100$ compared to the case where one marginalises over their (extremely wide) prior (see their Table 2). The gain on the constraints on dark energy parameters alone is instead only mild: a factor 2 . The precise computation of the gains in our specific case is, however, outside the aim of this work.

\section{Summary}

The aim of this work was threefold: first, using 53 clusters with individual measurements of mass, we derived the richness-mass scaling in the local Universe. We found a $0.46 \pm 0.12$ slope and a $0.25 \pm 0.03$ dex scatter in $(\log )$ richness at a given mass measuring the richness following the Andreon \& Hurn (2010) prescriptions. The fit accounts for the fact that the cluster sample is X-ray selected and massive clusters are over-represented, although we found that the sample selection is a minor source of concern for this sample. Because the scatter around the regression is derived from measurements of the individual masses and richnesses, our measurement of the scatter is preferable to others derived without knowledge of individual cluster masses, such as those of the maxBCG team (e.g. Rykoff et al. 2011).

Second, using $2500.06<z<0.9$ clusters with spectroscopic redshift, mostly at $z<0.3$, we found that the cluster redshift can be derived with an accuracy better than $\Delta z=0.02$ from the colour of the red sequence.

Third, we computed the predicted prior between mass and richness, i.e. one of the input ingredients to judge how strongly future surveys using clusters may constrain cosmological parameters, and to which extent clusters can compete with other cosmological probes. 

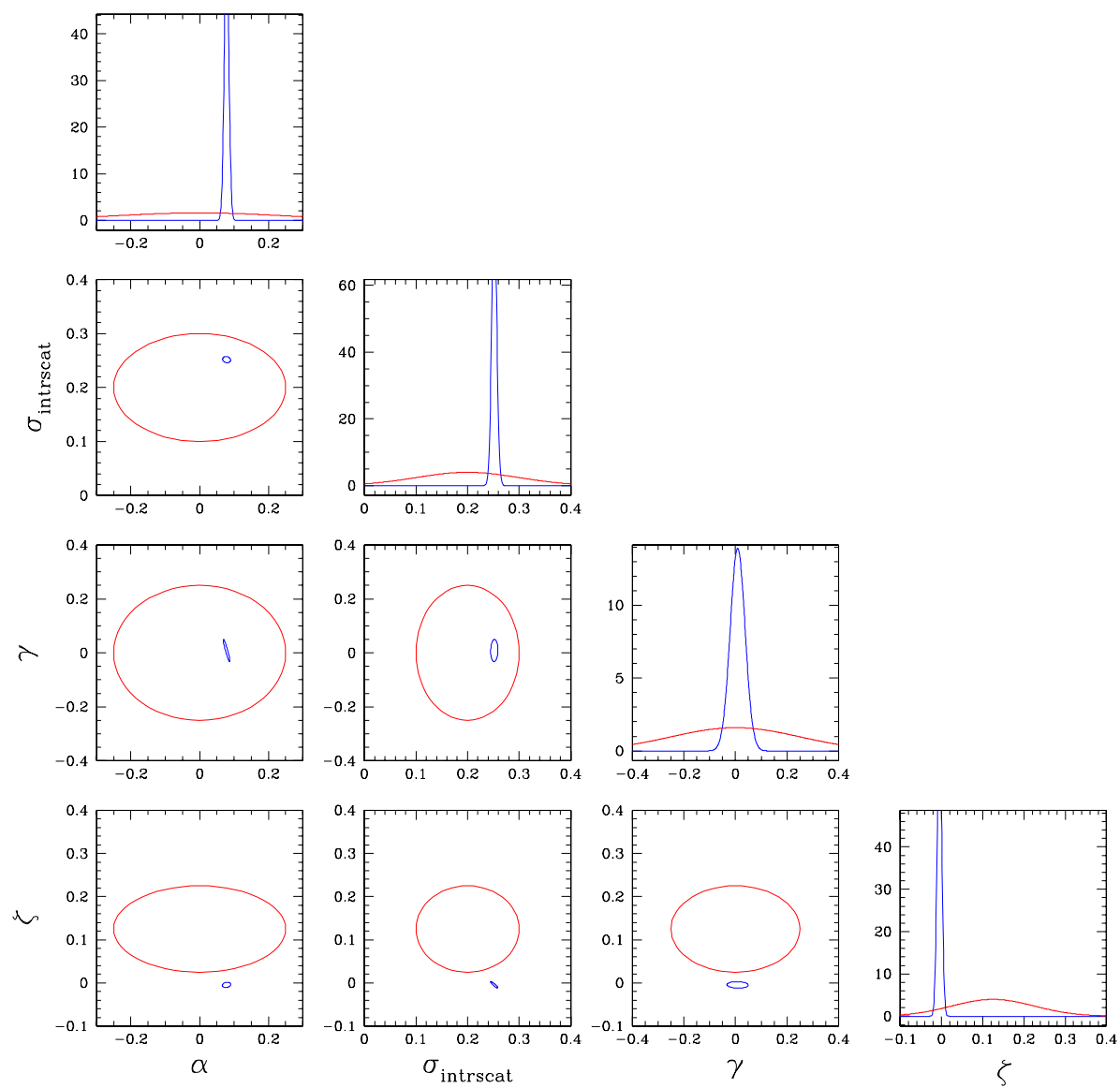

Fig. 10. Example of mismatch between predicted prior of the richness-mass scaling, as derived by us (in blue) and as adopted in other works (in red, from Carbone et al. 2012). This comparison should be seen as indicative only, because of differences between the two mass-proxy modellings.

To this aim, we generated a simulated universe obeying the derived richness-mass scaling, observed it with a mock PanStarrs 1+Euclid-like survey, allowing for intrinsic scatter between regressed quantities, allowing for mass and richness errors, and also allowing for cluster photometric redshift errors. The generated sample does not contain any cluster with an $S / N>5$ weak-lensing detection at $z>0.62$ (Fig. 7).

We fitted the observations with an evolving richness-mass scaling with five parameters to be determined. We allowed an evolution in the intercept (sometime called bias) and intrinsic scatter. We allowed an uncertainty on the intrinsic scatter and on the intercept, as previous works, but in contrast to all previous approaches, we did not sidestep the modelling of the slope.

Our fitting model recovers the input parameters, but only if the cluster mass function and the redshift-dependent $S / N>$ 5 weak-lensing survey selection function are accounted for. Neglecting them causes fit values to deviate by $>5 \sigma$ from the input values, as a result of the neglected Malmquist-like bias. This result emphasizes the limitations of often adopted simplifying assumptions, such as mass-complete redshift-independent samples. Including the optical selection function is unnecessary because all clusters with a weak-lensing signature are so massive and rich that detecting their red galaxy overdensity is trivial. Already available imaging data from PanStarrs 1 are of sufficient quality to detect these galaxies, whereas mass estimates await the Euclid mission.

We derived the uncertainty and the covariance matrix of the (evolving) richness-mass scaling, which are the input ingredients of every cosmological forecast using cluster counts. These five parameters will be known with percent precision thanks to masses estimated from Euclid data. There are non-negligible covariance terms between the five regression parameters. These numbers, listed in Table 1, are the third main result of this work. Their determination does not require the success, or acquisition, of other data presently not available, which is requested for other cluster surveys, such as the XXLS and SPT survey.

We found that the richness-mass scaling parameters can be determined $10^{5}$ better (the volume enclosed by the posterior is $10^{5}$ times smaller) than estimated before without using weaklensing mass estimates, although we emphasize that this number was derived using scaling relations with different parametrizations. A better knowledge of the scaling parameters likely has a strong impact on the relative importance of the different probes used to constrain cosmological parameters.

Finally, we checked that if the intrinsic scatter between mass and richness increases by a factor two by $z=0.6$, we are nevertheless able to recover the mass-richness scaling without bias, with only a factor 9 (about 1.5 per parameter) degradation in the quality with which we are able to recover the scaling parameters.

The fitting code, including of the treatment of the mass function and the weak-lensing selection function, is provided in the appendix. It can also be re-used, for example, to derive the predicted prior of other observable-mass scalings, such as the $L_{\mathrm{X}}$-mass relation.

Acknowledgements. We thank C. Carbone, B. Sartoris, and C. Strege for their comments on an early version of this draft and the referee for his/her request of clarifying our presentation concerning the impact of selection effects in the determination of the mass-observable scaling. 
S. Andreon and J. Bergé: Richness-mass relation self-calibration for galaxy clusters

\section{References}

Abbott, Aldering, G., Annis, J., et al. 2005, The Dark Energy Survey [arXiv:astro-ph/0510346]

Abell, G. O. 1958, ApJS, 3, 211

Akritas, M. G., \& Bershady, M. A. 1996, ApJ, 470, 706

Andreon, S. 2003a, A\&A, 409, 37

Andreon, S. 2003b, Astrophys. Space Sci., 285, 143

Andreon, S. 2012, A\&A, submitted

Andreon, S., \& Moretti, A. 2011, A\&A, 536, A37

Andreon, S., \& Hurn, M. A. 2010, MNRAS, 404, 1922

Andreon, S., \& Hurn, M. A. 2012, Statistics and Data Mining, submitted

Andreon, S., Trinchieri, G., \& Pizzolato, F. 2011, MNRAS, 412, 2391

Andreon, S., Willis, J., Quintana, H., et al. 2004a, MNRAS, 353, 353

Andreon, S., Willis, J., Quintana, H., et al. 2004b, in the proceeding of Exploring the Universe. Contents and Structure of the Universe, eds. Y. Giraud-Heraud,

J. Thanh Van, \& The Gioi, Vietnam [arXiv: astro-ph/0405574]

Bergé, J., Amara, A., \& Réfrégier, A. 2010, ApJ, 712, 992

Bruzual, G., \& Charlot, S. 2003, MNRAS, 344, 1000

Carbone, C., Fedeli, C., Moscardini, L., \& Cimatti, A. 2012, JCAP, 3, 23

Cuillandre, J.-C., \& Bertin, E. 2006, SF2A-2006: Semaine de l'Astrophysique Francaise, 265

Cunha, C. E., \& Evrard, A. E. 2010, Phys. Rev. D, 81, 083509

Gelman, A., Carlin, J., Stern, H., \& Rubin, D. 2004, Bayesian Data Analysis (Chapman \& Hall/CRC)

Gladders, M. D., \& Yee, H. K. C. 2000, AJ, 120, 2148

Hamana, T., Oguri, M., Shirasaki, M., \& Sato, M. 2012, MNRAS, 425, 2287

High, F. W., Stalder, B., Song, J., et al. 2010, ApJ, 723, 1736

Isobe, T., Feigelson, E. D., Akritas, M. G., \& Babu, G. J. 1990, ApJ, 364, 104

Jenkins, A., Frenk, C. S., White, S. D. M., et al. 2001, MNRAS, 321, 372

Johnston, D. E., Sheldon, E. S., Wechsler, R. H., et al. 2007, unpublished [arXiv:0709.1159]
Kodama, T., Arimoto, N., Barger, A. J., \& Arag'on-Salamanca, A. 1998, A\&A, 334, 99

Laureijs, R., Amiaux, J., Arduini, S., et al. 2011 [arXiv: 1110. 3193]

Lima, M., \& Hu, W. 2005, Phys. Rev. D, 72, 043006

Marian, L., \& Bernstein, G. M. 2006, Phys. Rev. D, 73, 123525

Navarro, J. F., Frenk, C. S., \& White, S. D. M. 1997, ApJ, 490, 493

Oguri, M., \& Takada, M. 2011, Phys. Rev. D, 83, 023008

Pace, F., Maturi, M., Meneghetti, M., et al. 2007, A\&A, 471, 731

Pacaud, F., Pierre, M., Adami, C., et al. 2007, MNRAS, 382, 1289

Pierre, M., Pacaud, F., Juin, J. B., et al. 2011, MNRAS, 414, 1732

Plummer, M., JAGS Version 2.2.0 user manual, 2010

Press, W. H., \& Schechter, P. 1974, ApJ, 187, 425

Puddu, E., Andreon, S., Longo, G., et al. 2001, A\&A, 379, 426

Refregier, A., Amara, A., Kitching, T. D., et al. 2010, The Euclid Imaging Consortium Science Book [arXiv: 1001.0061]

Reichardt, C. L., Stalder, B., Bleem, L. E., et al. 2012, ApJ, submitted [arXiv: 1203.5775]

Rines, K., \& Diaferio, A. 2006, AJ, 132, 1275

Rykoff, E. S., Koester, B. P., Rozo, E., et al. 2012, ApJ, 746, 178

Rozo, E., Rykoff, E. S., Evrard, A., et al. 2009, ApJ, 699, 768

Sartoris, B., Borgani, S., Fedeli, C., et al. 2010, MNRAS, 407, 2339

Shaw, L. D., Holder, G. P., \& Dudley, J. 2010, ApJ, 716, 281

Sheth, R. K., Mo, H. J., \& Tormen, G. 2001, MNRAS, 323, 1

Stanek, R., Evrard, A. E., Böhringer, H., Schuecker, P., \& Nord, B. 2006, ApJ, 648,956

Schechter, P. 1976, ApJ, 203, 297

Stanford, S. A., Eisenhardt, P. R., \& Dickinson, M. 1998, ApJ, 492, 461

Tinker, J., Kravtsov, A. V., Klypin, A., et al. 2008, ApJ, 688, 709

Thomas, D. B., \& Contaldi, C. R. 2011, JCAP, 12, 13 


\section{Appendix A: Model for computing the predicted prior of the mass-richness scaling including the weak-lensing selection function}

Equations (12) and (17) to (29) are almost literally translated into JAGS (Plummer 2008), Poisson, normal, and uniform distributions become dpois, dnorm, dunif, respectively. JAGS ${ }^{5}$, following BUGS (Spiegelhalter et al. 1995), uses precisions, prec $=1 / \sigma^{2}$, in place of variances $\sigma^{2}$. The only complication comes from sampling from a distribution unavailable in JAGS, a truncated Schechter function. This is achieved by exploiting the property that a Poisson $(\phi)$ observation of zero has a likelihood $e^{-\phi}$. Conseguently, if our observed data are a set of 0 's, and $\phi[i]$ is set to $-\log \mathcal{L}[i]$, we obtain the correct likelihood contribution. The quantity $\lambda[i]$ should always be greater than 0 , because it is a Poisson mean, and we may accordingly need to add a suitable constant, $C$, to ensure that it is positive. The quantity lg10tot. norm normalises the integral of the obslgM200 likelihood to one. The model (set of equations) reads in JAGS:



To adopt a Student $t$-distribution with ten degrees of freedom dt to model the intrinsic scatter (Sect. 4), it suffices to replace the line starting by $\operatorname{lgn} 200[i]$ with

$\operatorname{lgn} 200[\mathrm{i}] \sim \operatorname{dt}(\operatorname{lgn} 200 \mathrm{~m}[\mathrm{i}], \operatorname{prec}$.intrscat.z[i], 10)

\footnotetext{
5 http://calvin.iarc.fr/ martyn/software/jags/
} 